\title{
Analysis of Aboveground Vegetative Growth by Using the Logistic Theory of the Density Effect in Young Monocultures of Ficus carica $\mathbf{L}$.
}

\author{
Takuo Yamakura* ${ }^{1 *}$ Akihiro Hosomi ${ }^{2}$ and Daisuke Hirayama1 \\ ${ }^{1}$ Department of Biology, Graduate School of Science, Osaka City University, Osaka 558-8585, Japan \\ ${ }^{2}$ Agricultural, Food and Environmental Science Research Center of Osaka Prefecture, Osaka 583-0862, Japan
}

\begin{abstract}
The growth characteristics of the aboveground vegetative organs in two fig cultivars, 'Masui dauphine' and 'Houraishi' (Ficus carica L.), were analyzed by applying numerical techniques of logistic theory of the density effect to the results from our preceding study, which dealt with the influence of tree density on aboveground vegetative growth and reproduction in young fig monocultures. Three major growth characteristics, biological time $(\tau)$, growth rate $(\lambda)$, and final yield $(Y)$ in aboveground vegetative organs, were calculated by the theory, and their seasonal changes were examined. The $\tau$ and $Y$ represented a sigmoid curve with respect to time, while $\lambda$ peaked in late June or early July and decreased after mid-July. Throughout the growing season before defoliation, $\tau, \lambda$, and $\boldsymbol{Y}$ were always larger in 'Houraishi' than in 'Masui dauphine', clearly suggesting the superiority of 'Houraishi' to 'Masui dauphine' in aboveground vegetative growth. The proposed index could express the distance between focal cultivars in terms of three growth characteristics. By comparing $\lambda$ values of the cultivars with those of other herbs and trees, the status of cultivars in the biological gradient of plant growth rates was examined, and it was concluded that the cultivars are neither herbs nor trees but in-between. Furthermore, the effect of plant mass removal caused by natural or artificial events was incorporated into the reciprocal equation of the density effect in order to discuss leaf defoliation and shoot pruning.
\end{abstract}

Key Words: density, Ficus carica, 'Houraishi', logistic theory, 'Masui dauphine'.

\section{Introduction}

Figs (Ficus carica L.) in Japanese horticulture are characterized by remarkable vegetative growth, active nodal bud breaks, easy vegetative propagation using cuttings, sensitivity to cold temperature, deciduous traits in winter, strong demand for water and oxygen in roots, intolerance both to desiccation and bad drainage of soil water, inevitable soil sickness, short longevity of tree individuals, early beginning of fruit production, shortlife of mature fruit, and big differences in mature fruit qualities related with harvest timing (Hirata, 2000; Kabumoto et al., 1996; Sugiura et al., 2004). Of these characteristics, the vegetative growth of figs is a focal point in this study.

With respect to the various growth traits of figs, the increase of vegetative mass in an individual fig tree is expected to be well describable by a logistic equation because this equation has been widely used for the description of plant growth (Hozumi, 1973). It is a

Received; November 14, 2006. Accepted; May 2, 2007.

* Corresponding author (E-mail: yamakura@sci.osaka-cu.ac.jp). mathematical system that consists of two variables and two coefficients. The system variables are time $(t)$ and vegetative mass $(w)$, while the coefficients are the intrinsic growth rate $(\lambda)$ and carrying capacity $(W)$. Thus, the degree of freedom is four in the system. Since the two variables, $t$ and $w$, of the system are observable by monitoring plant growth at different times in actual plant cultivation, the residual degree of freedom in the system can become two; therefore, the final degree of freedom in the system can be decreased to one, if we can add a limiting condition either to $\lambda$ or $W$. It follows that the variables and coefficients of the system are explicitly determinable if we experimentally cultivate plants, control the growth factors and monitor plant growth at different timings. This theoretical consideration of plant growth and degree of freedom in the logistic equation offered a basis for derivation of the reciprocal equation of the C-D effect that was coined by Shinozaki and Kira (1956) (hereafter, the SK model). In this study, the logistic equation and SK model are fully adopted for analyzing the growth traits of aboveground vegetative organs in young fig trees.

In a preceding study (Yamakura et al., 2008), we 
described the outline of our spacing experiment in a fig orchard and confirmed the applicability of the SK model to explain the relationship between planted fig tree density and mean aboveground vegetative mass per tree per population. Since the two coefficients of $A$ and $B$ in the SK model are written as the mathematical functions of growth time $(t)$ and the coefficients, $\lambda$ and $W$, of the logistic equation, both $\lambda$ and $W$ can be obtained by the reverse calculation of coefficients of $A$ and $B$ with respect to $t$ (Shinozaki and Kira, 1956). Using the results from our former study, we calculated $\lambda$ and $W$, and compared two cultivars, 'Masui dauphine' (hereafter, 'Masui') (San Piero sensu Condit, 1955) and 'Houraishi' (conventional Japanese cultivar possibly from China), in terms of $\lambda$ and $W$ in this study. The conventional recognition of the superiority of 'Houraishi' to 'Masui' in vegetative growth is also numerically examined.

\section{Materials and Methods}

A major data source in this study are the results from our preceding study (Yamakura et al., 2008), which is briefly outlined here. Nursery stocks of 'Masui' and 'Houraishi' were raised by cutting. They were planted, changing the density of fig trees, and cultivated using straight line training in an experimental field in the Agricultural, Food and Environmental Science Research Center of Osaka Prefecture in 2004. The density grades per 1.0 -a $(10 \mathrm{~m} \times 10 \mathrm{~m})$ land area were $200,66.7,40$, and 6.06 , respectively. The spacing of fruit-bearing shoots (hereafter, shoots) along the primary scaffold limb (hereafter, limb) was $20 \mathrm{~cm}$ for all limbs. The shoots were not pruned and were allowed to grow in natural form in the growing season, although disbudding was carried out to exclude secondary shoots. All shoots were pruned on 21 March 2006. The growth of the aboveground vegetative parts of each tree was monitored at irregular intervals in 2005 and 2006 by measuring the length and diameter of three organs, i.e. main trunk, limb, and shoot. The monitored linear dimensions of the three organs were transformed into dry weight values of the respective organs and leaves by using numerical techniques in metrical geometry and allometry, and led to aboveground vegetative weight per tree as a sum of aboveground wood weight and leaf weight. Root weight was omitted in the weight computation because root sampling was impossible in growth monitoring (Yamakura et al., 2008).

The abovementioned aboveground vegetative weight per tree was tentatively designated either as individual fig tree weight or individual tree weight, and its mean $(w)$ in a population with given density $(\rho)$ was calculated. The dependency of $w$ on $\rho$ was approximated by the SK model, which is written as (Hirano, 1989; Kuroda et al., 1996; Shinozaki and Kira, 1965),

$$
1 / w=A \rho+B
$$

where $A$ and $B$ are coefficients specific to cultivars (or species) and time. The coefficients of Eq. (1), which were determined using a computer soft-ware package (DeltaGraph ver. 4.5, Japan Poladigital, Japan) on a Macintosh platform in the preceding study, are the major source data in this study and are reproduced in Table 1.

The two coefficients in Table 1 were analyzed, following the logistic theory of the density effect (synonymous with the logistic theory of the C-D effect; Shinozaki and Kira, 1956) or logistic theory of plant growth (Shinozaki, 1961), of which relevant essentials are reproduced below. In the theory, the logistic equation is written as

$$
\begin{aligned}
& \frac{d w}{d t}=\lambda(t) w\left(1-\frac{w}{W(t)}\right), \\
& w=\frac{e^{\tau}}{\int_{0}^{\tau} \frac{e^{\tau}}{W} d \tau+\frac{1}{w_{0}}} \\
& \tau=\int_{0}^{t} \lambda(t) d t, \\
& \left.w_{0}=w\right)_{t=0}
\end{aligned}
$$

where the variable $\tau$ is a dimensionless quantity representing biological time (Hozumi, 1977; Shinozaki, 1961), $w_{0}$ results from an integration constant in the solution of the differential equation of Eq. (2) and stands for the initial value of $w$ at $t=0$, and the other symbols follow the definitions already described. It is noteworthy that the coefficients of the logistic equations, $\lambda$ and $W$, are changeable with respect to $t$ and are expressed by the implicit functions of $t$ (Shinozaki and Kira, 1956).

The SK model of Eq. (1) is derived by combining Eqs. (2)-(5) with an important agronomic phenomenon, the law of constant final yield (Shinozaki and Kira, 1956), which is written as

$$
W(t) \rho=Y(t) \text { or } W(t)=Y(t) / \rho
$$

where $Y(t)$ is the constant final yield specific to cultivars and time and takes a fixed value for all $\rho$ when $t$ is concrete. The yield represents the dry weight of aboveground vegetative organs in unit land area and

Table 1. Coefficients of the SK model applied to aboveground vegetative growth in fig trees.

\begin{tabular}{lrcccc}
\hline \hline \multirow{2}{*}{$\begin{array}{l}\text { Date in } \\
\text { monitoring }\end{array}$} & \multicolumn{2}{c}{ 'Masui dauphine' } & & \multicolumn{2}{c}{ 'Houraishi' } \\
\cline { 2 - 3 } \cline { 5 - 6 } & $\begin{array}{c}A \times 10^{-6} \\
{[\mathrm{a} / \mathrm{g}]}\end{array}$ & $\begin{array}{c}B \times 10^{-3} \\
{[1 / \mathrm{g}]}\end{array}$ & & $\begin{array}{c}A \times 10^{-6} \\
{[\mathrm{a} / \mathrm{g}]}\end{array}$ & $\begin{array}{c}B \times 10^{-3} \\
{[1 / \mathrm{g}]}\end{array}$ \\
\hline 21 Apr. 2005 & 102.50 & 5.2050 & & 38.47 & 7.6510 \\
3 Jun. 2005 & 67.13 & 2.9850 & & 23.02 & 3.5590 \\
7 Jul. 2005 & 19.10 & 0.5191 & & 6.889 & 0.4871 \\
18 Aug. 2005 & 12.46 & 0.3303 & & 4.893 & 0.2511 \\
20 Sep. 2005 & 9.44 & 0.1568 & & 4.461 & 0.1567 \\
19 Oct. 2005 & 9.42 & 0.1315 & & 4.016 & 0.1280 \\
21 Feb. 2006 & 10.46 & 0.1356 & 6.011 & 0.1298 \\
2 Mar. 2006 & 43.49 & 0.1483 & 25.66 & 0.1591 \\
\hline
\end{tabular}


excludes fruit yield in this study. The coefficients of Eq. (1) are written as mathematical functions of variables and coefficients of Eqs. (2)-(6), i.e.

$$
A=e^{-\tau} \int_{0}^{\tau} \frac{e^{\tau}}{Y} d \tau \text { and } B=\frac{e^{-\tau}}{w_{0}},
$$

where $Y=Y(t)$ in an abbreviated expression. In turn, the coefficients of the logistic equation are calculated by inversely solving Eq. (7) with respect to $\lambda$ and $W$ (Shinozaki and Kira, 1956). We adopted the numerical approximation expressions proposed by Shinozaki and Hozumi (1962) in solving the coefficients, $\tau, \lambda$, and $W$, i.e.

$$
\begin{aligned}
& \tau=-\ln \left(w_{0} B\right) \\
& \lambda=\lambda(t)=\frac{1}{t_{2}-t_{1}} \ln \frac{B_{1}}{B_{2}} \\
& Y=Y(t)=W(t) \rho \approx \frac{1-w_{0} B}{A}
\end{aligned}
$$

where Eq. (8) is an exact transformation of the second equation in Eq. (7), the symbols of $t_{1}$ and $t_{2}\left(t_{2}>t_{1}\right)$ in Eq. (9) stand for different times, and the alphabetical symbols, $B_{1}$ and $B_{2}$, in Eq. (9) are coefficient $B$ 's values of Eq. (1) at $t_{1}$ and $t_{2}$, respectively. The repeated explanations for other symbols in Eqs. (8)-(10) are abbreviated here. The growth coefficients, $\tau, \lambda$, and $Y$, were calculated by substituting $A$ and $B$ in Table 1 into Eqs. (8)-(10) in this study.

\section{Results}

\section{Calculated growth characteristics of the logistic equation}

The coefficients, $\tau, \lambda$, and $Y$ as an alternative to $W$, of the logistic equation and SK model were designated the growth characteristics or traits (Shinozaki and Kira, 1956). Three growth characteristics were calculated using Eqs. (8)-(10). In the calculation, the initial tree weight of $w_{0}$ was $8.92 \mathrm{~g}$ in dry weight in 'Masui' and $6.65 \mathrm{~g}$ in 'Houraishi'. These $w_{0}$ values were equivalent to the dry weight of a cutting with a length of $10 \mathrm{~cm}$ in raising nursery stocks. Although the cutting was $20 \mathrm{~cm}$ in total length in two cultivars, its aboveground length was $10 \mathrm{~cm}$ on average. The time of growth monitoring represented tentative days after 31 March 2005 because fig growth was first monitored in April 2005. The unit of final yield $Y$ was DW $\mathrm{kg}$ per 1.0-a land area. The SK model was empirically available for describing the $\rho$ vs. $w$ relation in the winter, when the leaves and shoots were deprived by defoliation and shoot pruning, respectively. The major defoliation was initiated around mid-November and continued $c a$. one month. However we confined the computation of growth characteristics only to the growing season before defoliation since the SK model did not confirm the numerical techniques for analyzing the characteristics at the time of severe plant mass removal. The growth characteristics values thus calculated are listed in Table 2.

Change of growth characteristics with respect to time

Biological time $(\tau)$ increased with the increase of time $(t)$, and its trajectory with respect to $t$ followed a sigmoid curve within the growing season from spring to autumn. The progress of $\tau$ in 'Houraishi' was more rapid than that in 'Masui', reflecting active vegetative growth in 'Houraishi' (Fig. 1).

The derivative of $\tau$ with respect to $t$ gives a relative growth rate $\lambda$ at $w<W=Y / \rho$. The coefficient $\lambda$ peaked around the end of June or beginning of July, sharply

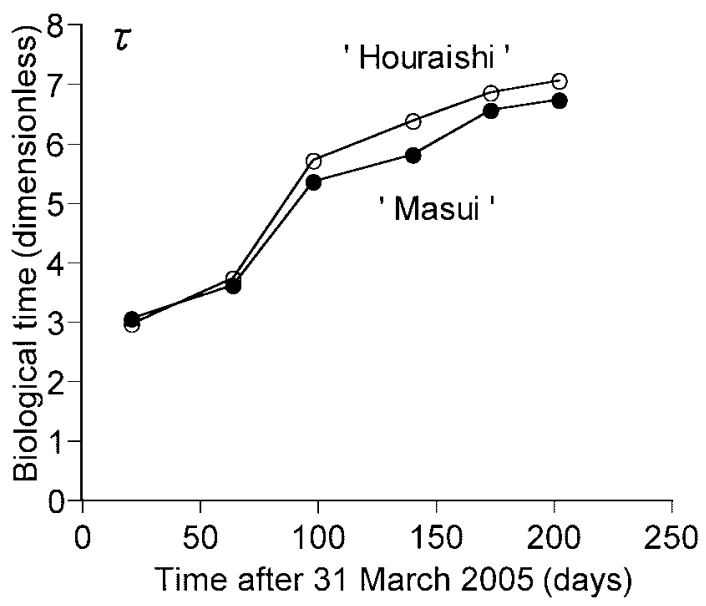

Fig. 1. Transition of $\tau$ with the progress of time after 31 March 2006 in the growth monitoring of aboveground vegetative organs in two fig cultivars. Closed circles and clear circles represent

\begin{tabular}{|c|c|c|c|c|c|c|}
\hline \multirow{2}{*}{$\begin{array}{l}\text { Days after } \\
31 \text { March } t\end{array}$} & \multicolumn{3}{|c|}{ 'Masui dauphine' } & \multicolumn{3}{|c|}{ 'Houraishi' } \\
\hline & $\tau$ & $\lambda \times 10^{-3 *}[1 /$ day $]$ & $Y[\mathrm{~kg} / \mathrm{a}]$ & $\tau$ & $\lambda \times 10^{-3 *}[1 /$ day $]$ & $Y[\mathrm{~kg} / \mathrm{a}]$ \\
\hline 21 & 3.070 & & 9.303 & 2.978 & & 24.672 \\
\hline 64 & 3.626 & 12.93 & 14.500 & 3.744 & 17.80 & 42.412 \\
\hline 98 & 5.375 & 51.45 & 52.114 & 5.732 & 58.49 & 144.689 \\
\hline 140 & 5.827 & 10.76 & 80.020 & 6.395 & 15.78 & 204.032 \\
\hline 173 & 6.572 & 22.58 & 105.784 & 6.867 & 14.29 & 223.931 \\
\hline 202 & 6.748 & 6.07 & 106.066 & 7.069 & 6.98 & 248.792 \\
\hline
\end{tabular}
'Masui dauphine' and 'Houraishi', respectively, in a species of Ficus carica L.

Table 2. Growth characteristics of logistic theory in two cultivars.

* Numerals in the i-th row correspond to the duration between $t$ in the $\mathrm{i}$-th row and $t$ in the (i-1)-th row. 
dropped in August, and gradually decreased in autumn. Although the general seasonal pattern of $\lambda$ was common in the two cultivars, slight recovery of $\lambda$ was observed in early autumn in 'Masui' (Fig. 2).

The final yield $Y$ increased with $t$ throughout the growing season. The seasonal patterns for the two cultivars, respectively, were similar to those in $\tau$, representing sigmoid curves with respect to $t$. The peak value of $Y$ in late autumn in 'Houraishi' was ca. 2.5 times greater than that in 'Masui' (Fig. 3).

\section{Comparison of two cultivars}

The growth traits, $\tau, \lambda$, and $Y$, can be strictly and easily compared between cultivars when the traits are simultaneously observable. If the time of growth trait observation is different between cultivars, the comparison becomes complicated because we should estimate
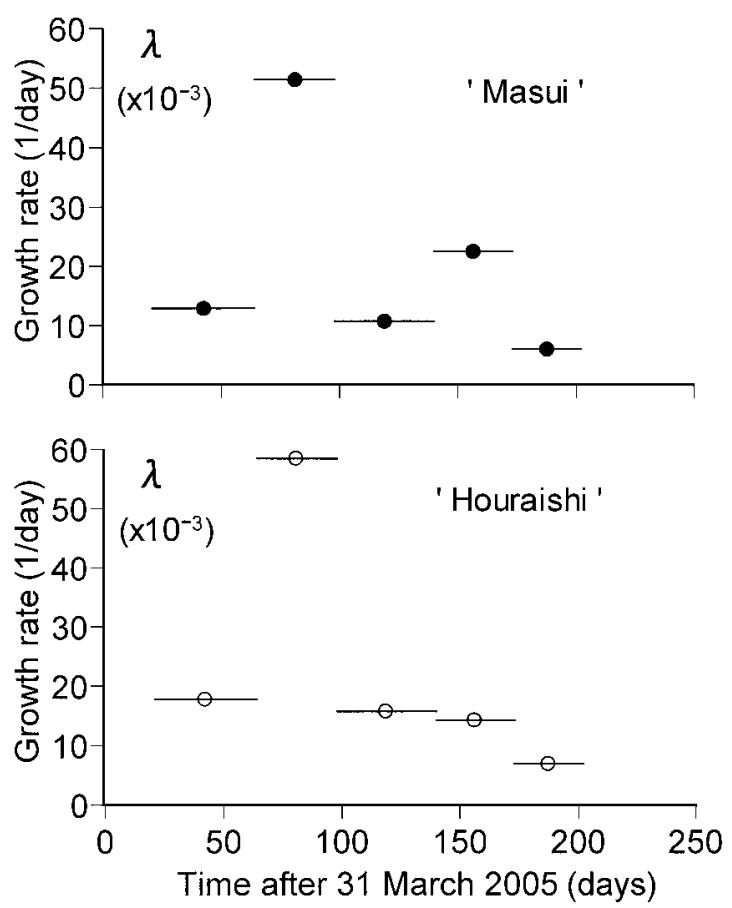

Fig. 2. Change of relative growth rates $\lambda$ in the course of aboveground vegetative growth in two fig cultivars. Closed and clear circles represent 'Masui dauphine' and 'Houraishi', respectively. the change of the traits with respect to time. Our results satisfied the conditions required for the easy comparison of growth traits between cultivars. In our comparison, the growth trait of 'Masui' at given time $t_{1}$ was contrasted with that of 'Houraishi' at $t_{1}$. 'Houraishi' was 1.054 , 1.086 and 2.348 times greater in terms of $\tau, \lambda$, and $Y$, respectively, than 'Masui'. These ratios clearly explained the superiority of 'Houraishi' to 'Masui' in vegetative growth (Fig. 4). Furthermore, the ratios led to a proposed measure for the relative Euclidian distance per growth trait between cultivars, i.e.

$$
Z=\sqrt{\left(\left(\tau_{i} / \tau_{j}-1\right)^{2}+\left(\lambda_{i} / \lambda_{j}-1\right)^{2}+\left(Y_{i} / Y_{j}-1\right)^{2}\right) / 3}
$$

where symbol $Z$ represents the proposed measure, subscripts $i$ and $j$ stand for 'Houraishi' and 'Masui', respectively, and the denominator of three in the root sign represents the number of focal growth traits. Relative trait ratios are preferable because we summed the trait differences. $Z$ is always positive and takes the value of 0.0 when 'Houraishi' is identical with 'Masui' in respective growth characteristics. By substituting the aforementioned three ratios into Eq. (11), we get $Z=$

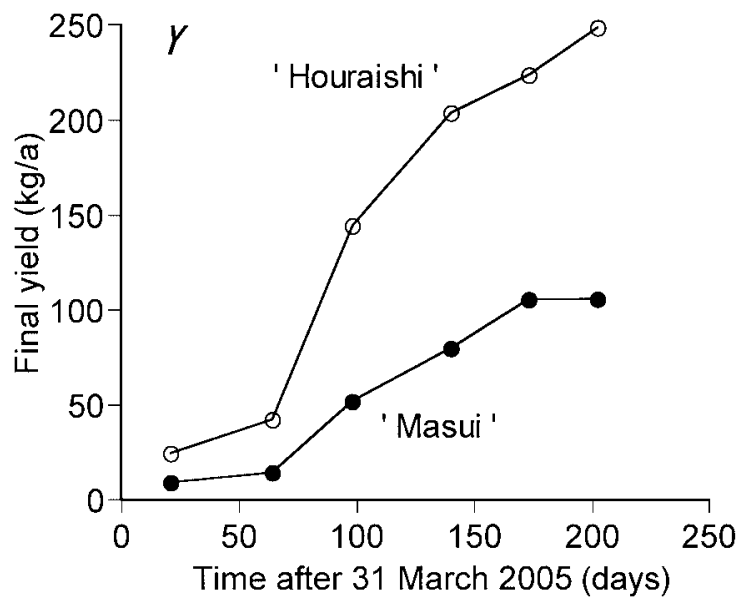

Fig. 3. Shift of the final yield $Y$ in aboveground vegetative organs through growth monitoring duration. Closed and clear circles represent 'Masui dauphine' and 'Houraishi', respectively.
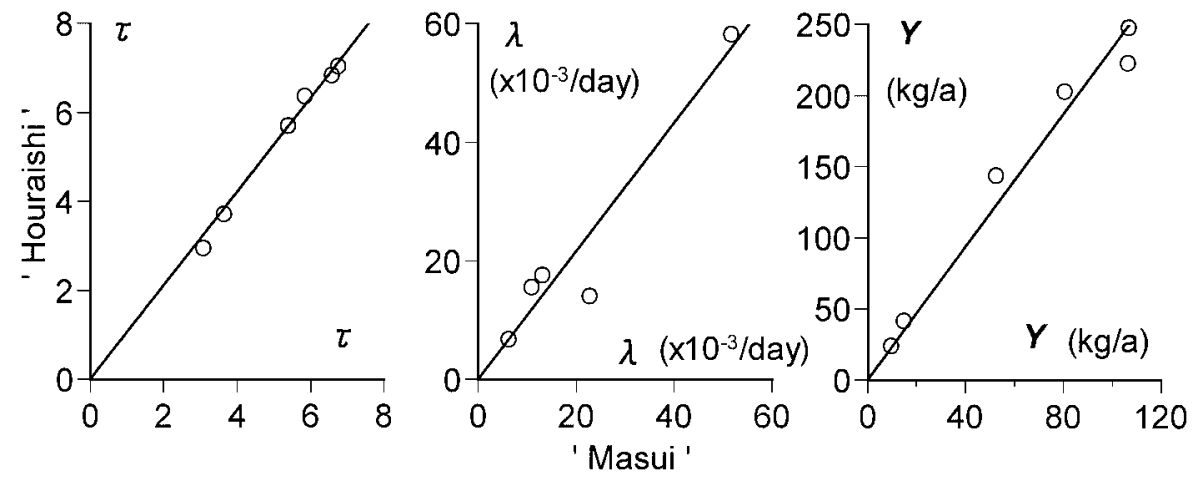

Fig. 4. Comparison of 'Masui dauphine' and 'Houraishi' in terms of three growth characteristics, $\tau$, $\lambda$, and $Y$. 
0.78 , although it is unfortunately impossible to refer to any comparable data. The $Z$ is also calculable in different seasons if the seasonal changes of three growth characteristics are given (Figs. 1-3). In our data, $Z$ was 1.13 in June, gradually decreased with the passage of time, reached a nadir in September $(Z=0.53)$ and increased again in October $(Z=0.78)$. The seasonal differences in $Z$ were mainly caused by differences in $Y$, suggesting the earlier initiation of vegetative growth and tolerance to cold in 'Houraishi'.

\section{Discussion}

\section{Final yield}

In the SK model, the law of constant final yield or constant final yield rule is an important point at which the logistic equation for plant growth is combined with plant density (Shinozaki, 1961; Shinozaki and Kira, 1956). The growth characteristic $Y$ represents the final yield common to different populations with different densities. In fig tree cultivation, $Y$ is a quantity that is annually accumulated in a tree body even if shoots and leaves are deprived. Therefore, its observed values for two fig cultivars should not be simply compared with those for annuals, for which several estimates of $Y$ were reported (Shinozaki and Kira, 1956). We have no comparable data of $Y$ for forest trees, although the SK model was applied to the descriptions of density effects in trees in forestry (Ando, 1968; Tadaki, 1963). Hirano (1989) reviewed his plentiful data on the density effect in orange groves. Kuroda et al. (1996) also compiled excellent data with respect to the density effect in apple trees, however, direct comparisons of $Y$ between fig trees and other fruit trees are not easy at present because the change of $w$ with respect to time is monitored on an annual basis in orange and apple trees. We should calculate the annual change of $Y$ in figs by continuing the experiment. The scarcity of available comparable data obliged us to tentatively compare $Y$ values of figs with annual or perennial herbs.

Since the fig trees were planted on 8 July 2004, the growth characteristics on 7 July 2005 in Tables 1 and 2 represent the quantities for one-year-old trees. The values of $Y$ for one-year-old fig populations are $52 \mathrm{~kg} \mathrm{DW} / \mathrm{a}$ in 'Masui' and $145 \mathrm{~kg}$ DW/a in 'Houraishi', respectively (Table 2). These $Y$ values of figs are rather similar to those of mature annuals, such as soybean $(Y=74.5 \mathrm{~kg}$ DW/a) (Shinozaki and Kira, 1956) and portulaca ( $Y=$ $48.8 \mathrm{~kg} \mathrm{DW} / \mathrm{a}$ ) (Ikushima et al., 1957), but far smaller than that of perennial rose mallow ( $Y=6431 \mathrm{~kg}$ DW/a) (Hozumi et al., 1958) grown in a cultivation pot of $11 \mathrm{~cm}$ in upper diameter, $7.5 \mathrm{~cm}$ in bottom diameter and $15 \mathrm{~cm}$ in depth. Since these $Y$ values for herbs include the root mass, the extraordinary magnitude of $Y$ in the perennial rose mallow may result from the large contribution of roots to $Y$, if the spacing per plant is properly evaluated. In plant cultivation using flowerpots, critical evaluation of the available space per plant is not easy because the aboveground parts of a mature plant tend to stick out of the pot.

\section{Neither a herb nor a tree}

The difference of $\tau$ or $\lambda$ between 'Masui' and 'Houraishi' was not as large as that of $Y$ (Fig. 4). The maximum daily growth rates of $\lambda$ were $0.0515 /$ day in 'Masui' and 0.0585/day in 'Houraishi', respectively (Table 2). The maximum $\lambda$ values of figs are compared with those of the aforementioned herbs, i.e, 0.159/day in soybean, $0.197 /$ day in portulaca and 0.239 /day in rose mallow (Hozumi et al., 1958; Ikushima et al., 1957; Shinozaki and Kira, 1956). The rose mallow's $\lambda$ is conspicuous and ca. 4.0 times greater than that of 'Houraishi'. Smaller $\lambda$ in figs may reflect the impact of the exclusion of roots on the computation.

Although $\lambda$ is obscure with respect to tree weight in forestry researches, the annual change of $\lambda$ in a stem volume unit was calculated for the western white pine by Hozumi (1977). His calculated largest $\lambda$ of the focal pine was $0.696 /$ year $=0.00191 /$ day, which appears to simulate an estimate of $\lambda$ in a weight unit because stem volume is proportional to stem weight. The $\lambda$ for the pine is far smaller than that of 'Masui'. The $\lambda$ values of stem diameters for various wild trees in temperate Japan and tropical Malaysia did not exceed $0.5 \mathrm{~cm} / \mathrm{cm} /$ year (Yamakura, 1996). This maximum $\lambda=\lambda(D)$ in stem diameters is hypothetically transformable into $\lambda=\lambda(w)$ in a weight unit by multiplying $\lambda(D)$ by an allometric constant, $h$, which connects the individual tree weight $(w)$ with stem diameters $(D)$ through the allometric equation of $w=a D^{h}$. The $h$ was within a range between 1.50 and 3.19 and the average was 2.40 (Ogawa and Kira, 1977). Applying the tentative numerical transformation to $\lambda(D)=0.5 \mathrm{~cm} / \mathrm{cm} /$ year for stem diameters, we get the estimate of $\lambda(w)=2.40 \times 0.5=1.20 /$ year $=0.00329 /$ day for an annual or a daily increase of tree weight. The indirect estimator of $0.00329 /$ day in wild trees suggests the tremendous growth potentials of figs among trees, though the large $\lambda(w)$ of fig trees resulted from a fertile orchard that is intensively managed. In a hypothetical conclusion from the comparison of $\lambda(w)$ values, the figs in this study are neither trees nor herbs but in-betweens in terms of $\lambda$.

\section{'Masui'vs. 'Houraishi'}

'Masui' was clearly inferior to 'Houraishi' in terms of $\tau, \lambda$, and $Y$ in aboveground vegetative growth, although the former was superior to the latter in annual fruit production (Yamakura et al., 2008). Thus the cultivar's ranking in vegetative growth reverses in reproduction, implying a tradeoff between vegetative growth and reproduction. The increase of fruit mass per tree with respect to time should be similarly analyzed by using the SK model to fully understand the tradeoff. Furthermore, cultivar response to different growth factors should also be studied in the future because we 
have dealt with the density effect alone in a variety of growth traits of focal cultivars and because a cultivar or species is recognized as a set of growth parameters, $\tau$, $\lambda$, and $Y$, in logistic theory (Shinozaki, 1961), which predicted the different behaviors of plants with respect to various growth factors including sick soil (Hosomi and Uchiyama, 1998; Hosomi et al., 2002). The concept of the growth parameter set as a cultivar or a species in the theory suggested the proposed measure of the relative Euclidian distance between cultivars $(Z)$, which is also applicable to another data set of plant traits, as described below.

Matsui (1989) reviewed the activities of leaf photosynthesis in fruit trees and listed species differences among eight fruit tree species in terms of three photosynthetic traits, the light compensation point, light saturation point, and photosynthesis rate at the light saturation point, respectively. Since his list included our focal fig cultivar of 'Masui', $Z$ was calculated by assigning 'Masui' for a base species $j$ and the other species for a pair species $i$. The $Z$ between 'Masui' and a 'Nankan-4' orange tree was 0.22 , while it was 0.31 between 'Masui' and a 'Fuji' apple tree. It was largest in a 'Tsukuba' Japanese chestnut tree $(Z=1.15)$. Unfortunately, $Z$ between 'Masui' and 'Houraishi' was unknown because 'Houraishi' was not covered by his list. The proposed $Z$ should be checked in further studies by continuing fig tree cultivation.

\section{SK model in plant mass removal}

In our fig orchard, leaves and shoots were removed from tree bodies by natural defoliation and artificial shoot pruning in winter. If plant mass removal in winter can be considered to simulate negative aboveground vegetative growth in natural conditions and if the $\rho$ vs. $w$ relation in winter can be approximated by the SK model, we may calculate three growth traits, $\tau$, $\lambda$, and $Y$, by using the numerical methods so far described; however, we did not calculate three growth traits in winter in this study, though the SK model was supported by the observed data. Hence, we tried to incorporate plant mass removal into the SK model and checked the possibility of growth trait calculation in winter.

Denoting the quantity of removed plant mass by the symbol $f(w, t)$, we rewrote Eq. (2) in the form of the following Eq. (12),

$$
\frac{d w}{d t}=\lambda(t) w\left(1-\frac{w}{W(t)}\right)-f(w, t)
$$

where $f(w, t)$ is an arbitrary function consisting of $w, t$, etc. and is determined by natural events, artificial horticultural steps for tree form management etc. Here we assume the very simple schedule of $f(w, t)=a(t) w$, where $f(w, t)$ is proportional to $w$, as described by Clark (1976) in fishery and Shinozaki (1976) in ecology. The proportional constant $a(t)$ is possibly less than 1.0 per unit $w$ per unit $t$ and changeable with respect to $t$, though it depends on the impact scales of natural events and tree form management plans in orchards. Substituting the proportional equation of $f(w, t)=a(t) w$ into Eq. (12), we get the relation,

$$
\begin{aligned}
& \frac{d w}{d t}=\lambda(t) w\left(1-\frac{w}{W(t)}\right)-a(t) w \\
& \frac{1}{w}=e^{-\tau} \int_{0}^{\tau} e^{\tau} \frac{\lambda}{\lambda-a} W d \tau+\frac{e^{-\tau}}{w_{0}}, \\
& \tau=\int_{0}^{t}(\lambda-a) d t,
\end{aligned}
$$

where Eq. (14) is the solution of Eq. (13) derived from Eq. (12), Eq. (15) explains the biological meaning of variable $\tau$ in Eq. (14), $w_{0}$ stands for initial weight of $w$ at $t=0$, and $\lambda=\lambda(t), \tau=\tau(t), a=a(t)$, and $W=W(t)$. Assuming the constant final yield rule and the constancy of $\rho$ with respect to $t$, and substituting Eq. (6) into Eq. (14), we can obtain a further SK model that has the same functional expression as Eq. (1), though its two coefficients should be rewritten by the equations,

$$
A=e^{-\tau} \int_{0}^{\tau} e^{\tau} \frac{\lambda}{\lambda-a} \frac{1}{Y} d \tau \text { and } B=\frac{e^{-\tau}}{w_{0}}
$$

where coefficient $a$ in the proportional plant mass removal is incorporated into the SK model. When $a<<\lambda$, Eq. (14), Eq. (15), and Eq. (16) correspond to Eq. (3), Eq. (4), and Eq. (7), respectively, therefore, the SK model can be adopted if $f(w, t)$ is either approximately proportional to $w$ or negligible small in terms of $a$. The calculation of $a, \lambda$, and $Y$ by inversely solving Eq. (15) and Eq. (16) with respect to time is omitted here because $\lambda$ and $a$ are inseparably combined with $\tau$ of Eq. (15) and make the calculation difficult, and because we regretfully did not measure $a=a(t)$ using practical horticultural steps in this study.

\section{Effect of tree form management on aboveground vegetative growth}

In fig orchards, a tree form is managed by applying a series of horticultural steps, such as the training of main trunks and scaffold limbs, pinching and pruning fruit-bearing shoots, disbudding secondary shoots in scaffold limbs and fruit-bearing shoots, rare fruit or leaf thinning, etc. These artificially steps tend to be combined with plant mass removal, may have a great influence on aboveground vegetative growth and may result in the peculiar tree growth that is impossible to be approximated by logistic equation, including Eq. (14). If this hypothetical speculation is true, the SK model should fail in explaining the results of spacing experiments in fruit trees in horticulture. Therefore, the consequences of this study might be built on the limited young growth stage of fig trees, in which the effect of plant mass removal for tree form management on fig tree growth is possibly negligible, and should be compared with further research results from older trees 
by continuing our spacing experiment.

\section{Acknowledgements}

The authors thank Dr. K. Hozumi (Emeritus Professor of Nagoya University) for his kind review of the manuscript.

\section{Literature Cited}

Ando, T. 1968. Ecological studies on the stand density control in even-aged pure stand. Bull. Gov. For. Exp. Stn., Tokyo, No. 210: 1-153 (In Japanese with English abstract).

Clark, C. W. 1976. Mathematical bioeconomics. John Wiley and Sons, New York.

Condit, I. J. 1955. Fig varieties: a monograph. Hilgardia 23: 323 538.

Hirano, S. 1989. Planting density (In Japanese). p. 156-201. In: S. Hirano and T. Kikuchi (eds.). Productivity and yields in fruit trees. Rural Culture Association, Tokyo.

Hirata, N. 2000. Growth characteristics of fig trees (In Japanese). p. 13-23. In: Rural Culture Association (ed.). Fig Encyclopedia of Fruit Science Vol. 13. Rural Culture Association, Tokyo.

Hosomi, A., M. Dan and A. Kato. 2002. Screening of fig varieties for rootstocks resistant to soil sickness. J. Japan. Soc. Hort. Sci. 71: 171-176.

Hosomi, A. and T. Uchiyama. 1998. Growth inhibiting factors in sick soil of fig orchards. J. Japan. Soc. Hort. Sci. 67: 44-50 (In Japanese with English abstract).

Hozumi, K. 1973. Plant interactions (In Japanese). Kyouritu Publishing, Tokyo.

Hozumi, K. 1977. Ecological and mathematical considerations on self-thinning in even-aged pure stands. I. Mean plant weightdensity trajectory during the course of self-thinning. Bot. Mag. Tokyo 90: 165-179.

Hozumi, K., T. Kira and K. Shinozaki. 1958. Effect of light intensity and planting density on the growth of Hibiscus moscheutos Lin., with special reference to the interaction between two linear factors of growth. Physiol. Ecol. 8: 36-
49 (In Japanese with English abstract).

Ikushima, I., T. Kira and K. Shinozaki. 1957. Growth curve analysis of Portulaca grandiflora Hook. Physiol. Ecol. 7: 98-112 (In Japanese with English abstract).

Kabumoto, T., K. Nakagawa, I. Irie, K. Yamashita and K. Matsu-ura. 1996. Fig (In Japanese). Rural Culture Association, Tokyo.

Kuroda, H., Y. Nishiyama and K. Chiba. 1996. Relationship between planting density and growth of 'Starking Delicious' apple trees grafted on dwarfing and semidwarfing rootstocks. J. Japan. Soc. Hort. Sci. 65: 227-236 (In Japanese with English abstract).

Matsui, H. 1989. Photosynthates and their distribution to different tree organs (In Japanese). p. 25-81. In: S. Hirano and T. Kikuchi (eds.). Productivity and yields in fruit trees. Rural Culture Association, Tokyo.

Shinozaki, K. 1961. Logistic theory of plant growth (In Japanese). $\mathrm{Ph}$. D. Thesis. Kyoto Univ., Kyoto.

Shinozaki, K. 1976. Effects of removal on a growing system. Physiol. Ecol. Japan. 17: 101-107 (In Japanese with English abstract).

Shinozaki, K. and K. Hozumi. 1962. An approximation of the coefficient $\mathrm{A}$ in the reciprocal equation. Physiol. Ecol. 11: 78-83 (In Japanese with English abstract).

Shinozaki, K. and T. Kira. 1956. Intraspecific competition among higher plants. VII. Logistic theory of the C-D effect. J. Inst. Polytech. Osaka City Univ. Ser. D.7: 35-72.

Sugiura, A., H. Koike, N. Kubota, F. Mizutani, K. Ban-no, M. Yamada and S. Taira. 2004. Fundamentals of fruit tree cultivation (In Japanese). Rural Culture Association, Tokyo.

Tadaki, Y. 1963. The pre-estimating of stem yield based on the competition-density effect. Bull. Gov. For. Exp. Stn., Tokyo, No. 154: 1-19 (In Japanese with English abstract).

Yamakura, T. 1996. Primary productivity and nutrient cycling in tropical rain forest (In Japanese). p. 258-291. In: G. Iwatsubo (ed.). Forest ecology. Buneido Publishing, Tokyo.

Yamakura, T., A. Hosomi and D. Hirayama, 2008. Effect of tree spacing on vegetative growth and reproduction in an early growth stage in two cultivars of Ficus carica L. J. Japan. Soc. Hort. Sci. 77: 7-16. 